\title{
HIPERURICEMIA Y PROGRESIÓN DEL COMPROMISO CARDIORRENAL CRÓNICO
}

\author{
Manuel A. Virú-Loza1,2,a, Alaciel Melissa Palacios-Guillén ${ }^{1,3, b}$
}

\begin{abstract}
RESUMEN
Con el objetivo de determinar si la hiperuricemia está asociada con la progresión del compromiso cardiorrenal crónico (CCC) se realizó un estudio de cohorte retrospectiva que incluyó a 103 pacientes en quienes se definió CCC como presencia simultánea de enfermedad renal crónica (ERC) y enfermedad cardiaca crónica (ECC). Se definió progresión de la ERC como la reducción del $50 \%$ de la tasa de filtración glomerular estimada por la ecuación MDRD-4. Se definió hiperuricemia como niveles de ácido úrico basal $\geq 7 \mathrm{mg} / \mathrm{dL}$. Ninguna de las variables estudiadas demostró asociación con la progresión del CCC o la ECC. Las variables que mostraron ser factores independientes de progresión de la ERC fueron hiperuricemia basal (HR 4,41; IC 95\%: 1,02-18,94) y PAS en el seguimiento (HR 1,05; IC 95\%: 1,01-1,09). Se concluye que la hiperuricemia mostró ser un factor de riesgo independiente de progresión de ERC en pacientes con CCC.
\end{abstract}

Palabras clave: Ácido úrico; Hiperuricemia; Enfermedad renal crónica; Síndrome cardiorrenal; Presión arterial; Estudios de seguimiento; Progresión de la enfermedad (fuente: DECS/BIREME).

\section{HYPERURICEMIA AND PROGRESSION OF CHRONIC CARDIORENAL COMPROMISE}

\begin{abstract}
In order to determine whether hyperuricemia is associated with the progression of chronic cardiorenal compromise (CCC), a retrospective cohort study was performed which included 103 patients in whom CCC was defined as the simultaneous presence of chronic kidney disease (CKD) and chronic heart disease (CHD). CKD progression was defined as a $50 \%$ reduction of glomerular filtration rate estimated by the MDRD-4 equation. Hyperuricemia was defined as basal levels of uric acid $\geq 7 \mathrm{mg} / \mathrm{dL}$. None of the studied variables showed association with the progression of CCC or CHD. Independent predictors of CKD progression were basal hyperuricemia (HR 4.41, 95\% Cl: 1.02-18.94) and SAP in followup (HR 1.05, 95\% Cl: 1.01-1.09). We conclude that hyperuricemia is an independent risk factor for the progression of CKD in patients with CCC.
\end{abstract}

Key words: Uric acid; Hyperuricemia; Renal insufficiency, chronic; Cardio-renal syndrome; Arterial pressure; Follow-up studies; Disease progression (source: MeSH/NLM).

\section{INTRODUCCIÓN}

A nivel global se ha observado un incremento en las tasas de enfermedad cardiaca, de enfermedad renal y la presencia concomitante de enfermedad cardiaca y renal, algunas cifras señalan que la enfermedad cardiaca estaría involucrada en más del $50 \%$ de las muertes de pacientes con enfermedad renal crónica (ERC), existe una sobreposición en la clínica y fisiopatología entre la ERC y la enfermedad cardiaca crónica (ECC) (1). Los niveles de ácido úrico estarían asociados con la progresión de la ERC ${ }^{(2-7)}$, por lo que la hiperuricemia puede desempeñar una función importante en la fisiopatología y en el pronóstico de la ECC (7-12).

Por lo expuesto, se puede entender la necesidad de realizar investigaciones sobre los factores de riesgo que estarían relacionados con el deterioro crónico de la

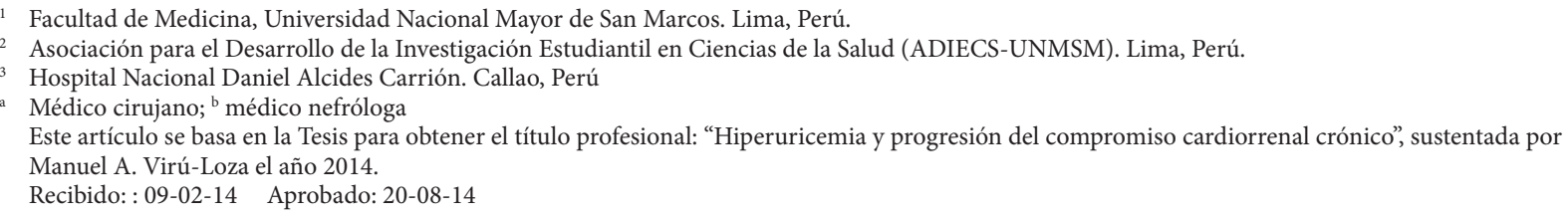

Citar como: Virú-Loza MA, Palacios-Guillén AM. Hiperuricemia y progresión del compromiso cardiorrenal crónico. Rev Peru Med Exp Salud Publica. 2014;31(3):530-34. 
función renal y cardiaca, y que, además, se encuentran en evaluación para ser utilizados en la práctica clínica como factores de riesgo modificables que permitan evitar la progresión de la enfermedad. Un ejemplo de estos factores es la hiperuricemia. Sin embargo, no hay que dejar de lado otros factores modificables importantes que ya forman parte de las estrategias de tratamiento para controlar el avance de la enfermedad y que, además, interactúan con otros factores, como lo es la presión arterial.

En este contexto, nace la necesidad de realizar estudios en pacientes que presentan compromiso renal y cardiaco simultáneo, y de estos, especialmente a aquellos con afección cardiorrenal crónica (presencia simultánea de ERC y ECC). En el presente estudio las formas de afección cardiorrenal crónica se agrupan bajo la denominación de compromiso cardiorrenal crónico (CCC). La importancia de abordar pacientes con CCC radica en que este tipo de afecciones son frecuentes en la práctica clínica, pero no son rutinariamente consideradas en el contexto de una unidad cardiorrenal. El objetivo del estudio es determinar si la hiperuricemia está asociada con la progresión del compromiso cardiorrenal crónico.

\section{EL ESTUDIO}

Estudio de cohorte retrospectiva Se estudió a todos los pacientes que presentaron CCC y que se atendieron por primera vez, entre enero de 2005 y diciembre de 2006, en el Servicio de Nefrología del Hospital Nacional Daniel Alcides Carrión en Perú. El límite del seguimiento fue julio de 2011. Se excluyó pacientes con gota, enfermedad renal congénita y enfermedad cardiaca congénita. Se obtuvo todos los datos a partir de las historias clínicas.

Se definió CCC como coexistencia de ERC y ECC. Se definió ERC como diagnóstico clínico por el médico tratante registrado en la historia clínica. ECC se definió como la evidencia expresa en la historia clínica de características concordantes con alguno de los estadios que plantea la American Heart Association. Se midió las siguientes variables: sexo, edad, tasa de filtrado glomerular obtenida con la ecuación MDRD-4, ácido úrico basal, presión arterial sistólica (PAS) basal, presión arterial diastólica (PAD) basal, PAS durante el seguimiento, PAD durante el seguimiento, evento cardiovascular previo (antecedente de accidente cerebrovascular, insuficiencia cardiaca descompensada o infarto agudo de miocardio), y diabetes mellitus tipo 2. Se definió hiperuricemia como ácido úrico $\geq 7 \mathrm{mg} / \mathrm{dL}$.

Para analizar los valores de presión arterial durante el seguimiento, primero se registró el mayor nivel evidenciado de cada tres meses, el cual se tomó como representativo de dicho intervalo. Luego, para obtener los valores de presión arterial en el seguimiento, se promedió los valores representativos de cada 3 meses. Para medir los indicadores de variabilidad de presión arterial se calculó la desviación estándar de los valores representativos de presión arterial de cada 3 meses. Se definió indicadores de progresión de ERC, ECC y de CCC. Se definió progresión de la ERC como reducción del $50 \%$ de la tasa de filtración glomerular estimada. Se definió progresión de la ECC como la aparición en la historia clínica de hospitalización por causa o evento cardiovascular luego de la fecha de inicio de seguimiento de cada paciente. Se definió progresión del CCC como un indicador compuesto por la aparición de progresión de la ERC o progresión de la ECC.

Los análisis estadísticos se realizaron utilizando el programa Stata 11.0. Las pruebas de chi cuadrado y t de Student fueron empleadas para comparar grupos de acuerdo con la presencia de hiperuricemia basal. Se utilizó modelos de riesgo proporcional de Cox para el análisis de la asociación entre la hiperuricemia y la progresión del CCC. El nivel de significancia fue de 0,05 . El protocolo del estudio obtuvo la aprobación del Comité de Ética del Hospital Nacional Daniel Alcides Carrión.

\section{RESULTADOS}

Se estudiaron un total de 103 pacientes. Las medidas descriptivas de las variables estudiadas para los grupos de pacientes con y sin hiperuricemia basal se encuentran en la Tabla 1. En la misma tabla también se muestra que la hiperuricemia basal estuvo asociada con una menor depuración de creatinina estimada inicial $(p<0,001)$, mayor PAS basal $(p<0,001)$, mayor PAD basal $(p=0,01)$, mayor PAS en el seguimiento $(p<0,001)$ y mayor $P A D$ diastólica en el seguimiento $(p=0,023)$.

Ninguna variable mostró asociación significativa con la progresión de la ECC y del CCC tanto en el análisis bivariado como en el multivariado (Tabla 2). Cabe resaltar que en ambos modelos multivariados, la variable PAD en el seguimiento no cumplió con el supuesto de riesgos proporcionales de Cox al utilizar el método de los residuos de Schoenfeld. Por tanto, los modelos que se muestran son resultado de un análisis estratificado para la variable PAD en el seguimiento.

Las variables que mostraron asociación con la progresión de la ERC en los modelos de riesgos proporcionales de Cox bivariados fueron: sexo femenino (HR 0,34; IC 95\%: 0,14-0,88), hiperuricemia basal (HR 
Tabla 1. Caracteríticas clínicas según nivel de ácido úrico

\begin{tabular}{|c|c|c|c|}
\hline & $\begin{array}{c}\text { Ácido úrico basal }<7 \mathrm{mg} / \mathrm{dL} \\
(\mathrm{n}=64)\end{array}$ & $\begin{array}{c}\text { Ácido úrico basal } \geq 7 \mathrm{mg} / \mathrm{dL} \\
(\mathrm{n}=39)\end{array}$ & Valor $p$ \\
\hline Niveles de ácido úrico & $5,1 \pm 1,4^{a}$ & $8,5 \pm 1,3^{a}$ & $<0,001^{d}$ \\
\hline Sexo femenino & $46(71,8 \%)$ & $21(53,8 \%)$ & $0,063^{c}$ \\
\hline Edad en años & $69,8 \pm 11,6^{a}$ & $69,7 \pm 12,7^{a}$ & $0,980^{d}$ \\
\hline Hiperuricemia basal & $21,9 \pm 16,7^{a}$ & $17,0 \pm 14,1^{a}$ & $0,133^{d}$ \\
\hline Tasa de filtrado glomerular basal & $64,9 \pm 29,1$ & $36,4 \pm 16,4$ a & $<0,001^{d}$ \\
\hline PAS inicial $(\mathrm{mmHg})$ & $130(120-150)^{b}$ & $150(130-160)^{b}$ & $<0,001^{e}$ \\
\hline PAD inicial $(\mathrm{mmHg})$ & $80(70-90)^{b}$ & $80(80-90)^{b}$ & $0,01^{e}$ \\
\hline PAS en el seguimiento $(\mathrm{mmHg})$ & $130(120,5-144,5)^{b}$ & $143,9(132,5-156,6)^{b}$ & $<0,001^{e}$ \\
\hline PAD en el seguimiento $(\mathrm{mmHg})$ & $77,3(72,15-82,9)^{b}$ & $80(75-88,3)^{b}$ & $0,023^{e}$ \\
\hline Evento cardiovascular previo & $15(23,4 \%)$ & $11(28,2 \%)$ & $0,589^{c}$ \\
\hline Accidente cerebrovascular & $5(7,8 \%)$ & $8(20,5 \%)$ & $0,060^{c}$ \\
\hline $\begin{array}{l}\text { Insuficiencia cardiaca } \\
\text { descompensada }\end{array}$ & $3(4,7 \%)$ & $1(2,6 \%)$ & $0,588^{c}$ \\
\hline Infarto agudo de miocardio & $1(1,6 \%)$ & $2(3,1 \%)$ & $0,297^{c}$ \\
\hline Diabetes mellitus diagnosticada & $22(34,4 \%)$ & $15(38,5 \%)$ & $0,675^{c}$ \\
\hline
\end{tabular}

PAS: presión arterial sistólica; PAD: presión arterial diastólica.

${ }^{\text {a }}$ Media \pm desviación estándar, ${ }^{\mathrm{b}}$ mediana (percentil 25 - percentil 75$),{ }^{\mathrm{c}}$ prueba de chi cuadrado, ${ }^{\mathrm{d}}$ prueba $\mathrm{t},{ }^{\mathrm{e}}$ prueba de suma de rangos de Wilcoxon

2,81; IC 95\%: 1,09-7,29), PAS en el seguimiento (HR 1,04; IC 95\%: 1,01-1,07) y PAD en el seguimiento (HR 1,06; IC 95\%: 1,01-1,12). Cuando se formuló el modelo de riesgo proporcional de Cox multivariado para evaluar la progresión de la ERC, las variables edad y PAD en el seguimiento no cumplieron con el supuesto de riesgos proporcionales de Cox. Por tanto, se utilizó un modelo de Cox estratificado para ambas variables dicotomizadas, en el cual se evidenció que las variables hiperuricemia basal (HR 4,41; IC 95\%: $1,02-18,94)$ y PAS durante el seguimiento (HR 1,05 ; IC 95\%: 1,01-1,09) tuvieron una asociación significativa Tabla 2.

\section{DISCUSIÓN}

La hiperuricemia, al igual que el resto de variables, no mostró asociación con la progresión del CCC o con la progresión de la ECC. La evidencia actual muestra que la hiperuricemia es un factor asociado a la progresión de enfermedad renal crónica y cardiaca crónica por separado y, por tanto, también debería estar asociada a la progresión de un indicador compuesto de progresión del compromiso cardiorrenal crónico. Sin embargo, en el presente estudio esto no se ha podido evidenciar, probablemente debido a no haber podido contar con un mejor indicador de progresión de enfermedad cardiaca crónica como el

Tabla 2. Modelos de riesgos proporcionales de Cox bivariado y multivariado

\begin{tabular}{|c|c|c|c|c|c|c|}
\hline & \multicolumn{2}{|c|}{ Progresión del CCC } & \multicolumn{2}{|c|}{ Progresión de la ECC } & \multicolumn{2}{|c|}{ Progresión de la ERC } \\
\hline & $\begin{array}{l}\text { HR bivariado } \\
\text { (IC } 95 \% \text { ) }\end{array}$ & $\begin{array}{l}\text { HR multivariado } \\
\text { (IC 95\%) }\end{array}$ & $\begin{array}{c}\text { HR bivariado (IC } \\
95 \% \text { ) }\end{array}$ & $\begin{array}{l}\text { HR multivariado } \\
\text { (IC 95\%) }\end{array}$ & $\begin{array}{c}\text { HR bivariado (IC } \\
95 \% \text { ) }\end{array}$ & $\begin{array}{l}\text { HR multivariado } \\
\text { (IC 95\%) }\end{array}$ \\
\hline Sexo femenino & $0,59(0,27-1,28)$ & $0,63(0,26-1,55)$ & $0,48(0,18-1,32)$ & $0,46(0,15-1,45)$ & $0,34(0,14-0,88)$ & $0,34(0,10-1,14)$ \\
\hline Edad en años & $0,99(0,96-1,02)$ & $0,98(0,94-1,02)$ & $0,99(0,95-1,03)$ & $0,98(0,92-1,03)$ & $0,99(0,95-1,03)$ & - \\
\hline Hiperuricemia basal & $1,73(0,81-3,71)$ & $2,10(0,77-5,71)$ & $1,19(0,432-3,32)$ & $1,34(0,35-5,14)$ & $2,81(1,09-7,29)$ & $4,41(1,02-18,94)$ \\
\hline $\begin{array}{l}\text { Tasa de filtración } \\
\text { glomerular basal }\end{array}$ & $0,99(0,98-1,01)$ & 1,00 (0,98-1,02) & $0,99(0,98-1,02)$ & 0,99 (0,97-1,02) & $0,99(0,97-1,01)$ & $1,01(0,98-1,04)$ \\
\hline PAS inicial (mmHg) & $1,00(0,99-1,02)$ & $0,99(0,96-1,02)$ & $1,00(0,98-1,03)$ & $0,99(0,96-1,03)$ & $1,01(0,99-1,03)$ & $1,00(0,96-1,04)$ \\
\hline PAD inicial (mmHg) & $1,01(0,98-1,04)$ & $0,99(0,94-1,04)$ & $1,00(0,97-1,05)$ & $0,98(0,92-1,05)$ & $1,03(0,99-1,07)$ & $0,96(0,90-1,02)$ \\
\hline $\begin{array}{l}\text { PAS en el } \\
\text { seguimiento }(\mathrm{mmHg})\end{array}$ & 1,01 (0,99-1,04) & $1,02(0,99-1,06)$ & $1,00(0,98-1,04)$ & $1,02(0,98-1,06)$ & $1,04(1,01-1,07)$ & $1,05(1,01-1,09)$ \\
\hline $\begin{array}{l}\text { PAD en el } \\
\text { seguimiento }(\mathrm{mmHg})\end{array}$ & $1,02(0,98-1,07)$ & - & $1,00(0,95-1,07)$ & - & $1,06(1,01-1,12)$ & - \\
\hline $\begin{array}{l}\text { Evento } \\
\text { cardiovascular previo }\end{array}$ & $0,83(0,33-2,08)$ & $0,82(0,30-2,20)$ & $0,48(0,11-2,13)$ & $0,52(0,11-2,49)$ & $0,86(0,28-2,64)$ & $0,96(0,23-3,92)$ \\
\hline
\end{tabular}

PAS: presión arterial sistólica; PAD: presión arterial diastólica. 
péptido natriurético atrial, lo cual afecta el desempeño del indicador de progresión de enfermedad cardiaca crónica y al indicador compuesto de compromiso cardiorrenal.

Por otro lado, tal y como se sabe actualmente, la hiperuricemia mostró asociación con la progresión de la ERC. En el presente estudio la variable "sexo femenino" actúa como un factor protector ante la progresión de la ERC, lo cual concuerda con algunas publicaciones, donde se menciona que en las mujeres los estrógenos tienen un efecto protector en el sistema cardiorrenal (13-15).

Los valores de presión arterial basal no muestran asociación alguna con la progresión de la ERC, probablemente porque una única medición de presión arterial carece de valor pronóstico. Esto también se ha visto en otros estudios ${ }^{(16)}$. Un régimen de disminución intensiva de la presión arterial provee protección contra eventos de deterioro de la ERC, particularmente en aquellos con proteinuria ${ }^{(17)}$. El presente estudio coincide en que presentar durante el seguimiento una PAS baja disminuye el riesgo de deterioro de la ERC.

Una variabilidad elevada de la presión arterial tampoco mostró asociación alguna, tanto en el análisis univariado como multivariado. Los resultados de estudios que han valorado la asociación entre la variabilidad de la presión arterial y la progresión de la ERC han sido contradictorios $(18,19)$, además, hay que tener en cuenta que no existe un indicador estándar para medir la variabilidad de la presión arterial y que, por este motivo, cada estudio plantea un indicador distinto. En el presente estudio la falta de asociación observada puede deberse a que el indicador utilizado no fue lo suficientemente preciso.

Niveles elevados de ácido úrico están asociados con una función renal deteriorada; si bien antes se planteó la pregunta de si esto se debe a que los niveles de ácido úrico son un indicador del estado de la función renal en un momento determinado o a que los niveles de ácido úrico elevados repercuten en la progresión de la enfermedad renal ${ }^{(20)}$, actualmente hay una amplia evidencia a favor de la hiperuricemia como factor de riesgo independiente de progresión de la ERC ${ }^{(2-5)}$. El presente estudio coincide en que aquellos pacientes con hiperuricemia presentan menores valores de depuración de creatinina, y a su vez la hiperuricemia muestra ser un factor de riesgo independiente de progresión de la ERC.

Entre las limitaciones del presente estudio está el no haber trabajado con una mayor cantidad de pacientes, utilizar una fuente de datos secundaria, el no haber establecido los grupos de pacientes de acuerdo a la presencia de hiperuricemia basal antes de comenzar el seguimiento, no haber podido contar con datos de microalbuminuria, y no haber podido contar con mediciones de marcadores de daño cardiaco como el péptido natriurético atrial.

Se puede concluir que la hiperuricemia mostró ser un factor de riesgo independiente de progresión de ERC en pacientes con CCC. Asimismo, que los resultados del presente estudio pueden generalizarse a los pacientes con compromiso cardiorrenal crónico que se atienden en el nosocomio en que se realizó esta investigación siendo necesario hacer estudios prospectivos a mayor escala en las poblaciones en que no existan datos suficientes sobre la progresión de la enfermedad cardiaca crónica y enfermedad renal crónica ya que la única forma-costo efectiva de abordar la carga de ERC es la prevención por medio de la modificación de factores de riesgo específicos para cada población.

Contribuciones de autoría: MVL y APG participaron en la concepción del artículo, la revisión crítica del artículo y aprobación de la versión final. MVL realizó la recolección de datos, el análisis e interpretación de resultados y la redacción del artículo.

Conflictos de interés: los autores declaran no tener conflictos de interés.

Financiamiento: autofinanciado

\section{REFERENCIAS BIBLIOGRÁFICAS}

1. Cruz DN, Bagshaw SM. Heart-kidney interaction: epidemiology of cardiorenal syndromes. Int J Nephrol. 2010 Dec 29;2011:351291. doi: $10.4061 / 2011 / 351291$

2. Bellomo G. Uric acid and chronic kidney disease: A time to act? World J Nephrol. 2013 May 6;2(2):17-25. doi: 10.5527/wjn.v2.i2.17.

3. Johnson RJ, Nakagawa T, Jalal D, Sánchez-Lozada LG, Kang DH, Ritz E. Uric acid and chronic kidney disease: which is chasing which? Nephrol Dial Transplant. 2013 Sep;28(9):2221-8. doi: $10.1093 /$ ndt/gft029.

4. Nashar K, Fried LF. Hyperuricemia and the progression of chronic kidney disease: is uric acid a marker or an independent risk factor? Adv Chronic Kidney Dis. 2012 Nov;19(6):386-91. doi: 10.1053/j. ackd.2012.05.004.

5. Zhu P, Liu Y, Han L, Xu G, Ran JM. Serum uric acid is associated with incident chronic kidney disease in middle-aged populations: a metaanalysis of 15 cohort studies. PLoS One. 2014 Jun 24;9(6):e100801. doi: 10.1371/journal.pone.0100801.

6. Minguela JI, Hernando A, Gallardo I, Martínez I, García P, Muñoz RI, et al. La hiperuricemia como factor de riesgo cardiovascular y renal. Dial Transpl. 2011 Abr-Jun;32(2):57-61.

7. Edwards NL. The role of hyperuricemia and gout in kidney and cardiovascular 
disease. Cleve Clin J Med. 2008 Jul;75 Suppl 5:S13-6.

8. Kanbay M, Segal M, Afsar B, Kang $\mathrm{DH}$, Rodriguez-Iturbe B, Johnson RJ. The role of uric acid in the pathogenesis of human cardiovascular disease. Heart. 2013 Jun;99(11):759-66. doi: 10.1136/heartjnl-2012-302535.

9. Gustafsson D, Unwin R. The pathophysiology of hyperuricaemia and its possible relationship to cardiovascular disease, morbidity and mortality. BMC Nephrol. 2013 Jul 29;14:164. doi: 10.1186/1471-236914-164.

10. Tamariz L, Harzand A, Palacio A, Verma S, Jones J, Hare J. Uric acid as a predictor of all-cause mortality in heart failure: a meta-analysis. Congest Heart Fail. 2011 Jan-Feb;17(1):25-30. doi: 10.1111/j.1751-7133.2011.00200.x.

11. Anker SD, Doehner W, Rauchhaus M, Sharma R, Francis D, Knosalla C, et al. Uric acid and survival in chronic heart failure: validation and application in metabolic, functional, and hemodynamic staging. Circulation. 2003 Apr 22;107(15):1991-7.

12. Hare JM, Mangal B, Brown J, Fisher C Jr, Freudenberger R, Colucci WS, et al. Impact of oxypurinol in patients with symptomatic heart failure. Results of the OPT-CHF study. J Am Coll Cardiol. 2008 Jun 17;51(24):2301-9. doi: 10.1016/j.jacc.2008.01.068.

13. Grzegorczyk K, Krajewska M, Weyde W, Jakuszko K, Gniewek A, Klinger M. [Gender and kidney diseases: the clinical importance and mechanisms of modifying effects]. Postepy Hig Med Dosw (Online). 2011 Dec 29;65:84957. [Article in Polish].

14. Carrero JJ. Gender differences in chronic kidney disease: underpinnings and therapeutic implications. Kidney Blood Press Res. 2010;33(5):383-92. doi: 10.1159/000320389.

15. Gava AL, Freitas FP, Meyrelles SS, Silva IV, Graceli JB. Gender-dependent effects of aging on the kidney. Braz J Med Biol Res. 2011 Sep;44(9):905-13.

16. Czernichow $S$, Zanchetti A, Turnbull F, Barzi F, Ninomiya T, Kengne AP, et al. The effects of blood pressure reduction and of different blood pressure-lowering regimens on major cardiovascular events according to baseline blood pressure: meta-analysis of randomized trials. J Hypertens. 2011 Jan;29(1):4-16. doi: 10.1097/ HJH.0b013e32834000be.

17. Lv J, Ehteshami P, Sarnak MJ, Tighiouart $\mathrm{H}$, Jun M, Ninomiya T, et al. Effects of intensive blood pressure lowering on the progression of chronic kidney disease: a systematic review and meta-analysis. CMAJ. 2013 Aug 6;185(11):949-57. doi: 10.1503/ cmaj. 121468 .

18. Di Iorio B, Pota A, Sirico ML, Torraca S, Di Micco L, Rubino R, et al. Blood pressure variability and outcomes in chronic kidney disease. Nephrol Dial Transplant. 2012 Dec;27(12):4404-10. doi: $10.1093 / \mathrm{ndt} / \mathrm{gfs} 328$.

19. Yokota K, Fukuda M, Matsui Y, Hoshide S, Shimada K, Kario K. Impact of visit-to-visit variability of blood pressure on deterioration of renal function in patients with non-diabetic chronic kidney disease. Hypertens Res. 2013 Feb;36(2):151-7. doi: 10.1038/ hr.2012.145.

20. Filiopoulos V, Hadjiyannakos D, Vlassopoulos D. New insights into uric acid effects on the progression and prognosis of chronic kidney disease. Ren Fail. 2012;34(4):510-20. doi: $10.3109 / 0886022 X .2011 .653753$.

Correspondencia: Manuel Virú Loza Dirección: Jr. Piedra Bigua 2241-A, San Juan de Lurigancho, Lima 36, Perú. Teléfono: (511) 3043417

Correo electrónico:m.andre.viru@hotmail.com

\section{Consulte las ediciones anteriores de la Revista Peruana de Medicina Experimental y Salud Pública en} www.scielosp.org

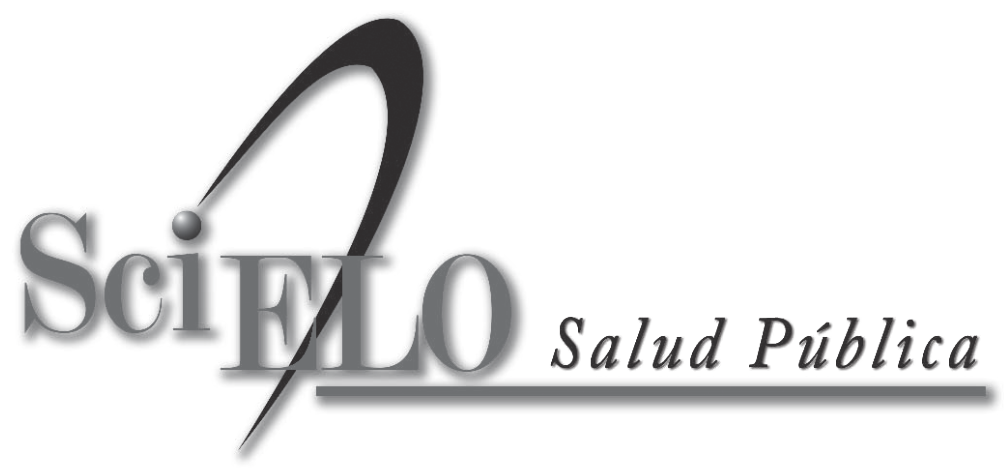

